\title{
Évaluation Comparative De La Qualité Nutritionnelle Et Sanitaire Du Lait De Différentes Races De Vaches De Quelques Zones D’élevage Du Bénin
}

\author{
Joseph Dossou \\ Département de Nutrition et Sciences Alimentaires, Faculté des Sciences \\ Agronomiques, Université d'Abomey-Calavi, Cotonou, Bénin \\ G. Donald Atchouké \\ Département de Biologie végétale, Faculté des Sciences et Techniques, \\ Université d’Abomey-Calavi, Cotonou, Bénin

\section{Sylvain Dabadé \\ Paulin Azokpota} \\ Jules Karel Montcho \\ Département de Nutrition et Sciences Alimentaires, Faculté des Sciences \\ Agronomiques, Université d’Abomey-Calavi, Cotonou, Bénin
}

doi: 10.19044/esj.2016.v12n3p141 URL:http://dx.doi.org/10.19044/esj.2016.v12n3p141

\begin{abstract}
In this study, a comparative assessment of the nutritional quality and the safety of milk from cow breeds Girolando, Borgou, Borgou-Gir, and Lagunaire produced in Benin was carried out in the public breeding centers of Kpinnou, Bétécoucou, Okparaand in private farms in Adjohoun, respectively. Milk from the breed Borgou-Gir had the highest concentrations of macronutrients (protein and lactose) and minerals (magnesium: $197.556 \pm$ $0.001 \mathrm{ppm}$, calcium: $2056.32 \pm 0.000 \mathrm{ppm}$, and zinc: $5.522 \pm 0.000 \mathrm{ppm}$ ). Analyzed milk samples were free of tuberculosis and brucellosis germs, and no mastitis prevalence was detected in milking cows. However, some milk samples had lead levels exceeding the maximum limit of $0.02 \mathrm{ppm}$ prescribed by the Codex Alimentarius standard. In addition, milk from the breeds Borgou and Borgou-Gir contained pesticide residue levels, namely chlorpyrifos $(0.12 \pm 0.020 \mathrm{ppm})$ and cypermethrin $(0.03 \pm 0.020 \mathrm{ppm})$ higher than the Codex Alimentarius standard (0.01 ppm). Milk samples from Adjohoun and Kpinnou had an aflatoxin M1 level of $0.3 \pm 0.000 \mathrm{ppb}$ and $0,010 \mathrm{ppb} \pm 0.24$, respectively and were not in agreement with the Codex Alimentarius standard $(0,05 \mathrm{ppb})$. Although rich in nutrients, analyzed cow's milk may present health risks to consumers due to chemical contaminants.
\end{abstract}


Keywords: Breed cattle, milk, safety, standards, chemical contaminants

\section{Résumé}

L'évaluation comparative de la qualité nutritionnelle et sanitaire du lait des vaches de races Girolando, Borgou, Borgou-Gir et Lagunaire produit au Bénin, a été effectuée respectivement dans les centres d'élevages de Kpinnou, Bétécoucou et Okpara et les élevages privés d’Adjohoun. Le lait des vaches de race Borgou-Gir avait les plus fortes teneurs en macronutriments (lactose et protéine) et en minéraux (magnésium: 197,556 $\pm 0,001$ ppm, calcium: 2056,32 $\pm 0,000$ ppm et zinc: 5,522 $\pm 0,000$ ppm). Les laits étaient exempts des germes de tuberculose, de brucellose et aucune prévalence de mammite n'était détectée chez les vaches traites. Par contre, certains échantillons de lait présentaient des taux de plomb dépassant la limite maximale de 0,02 ppm prévue par la norme du Codex Alimentarius. De plus, les laits des races Borgou et Borgou-Gir contenaient un taux de résidu de pesticide, notamment de chlorpyriphos-éthyl $(0,12 \pm 0,020 \mathrm{ppm})$ et de cyperméthrine $(0,03 \pm 0,020 \mathrm{ppm})$ dépassant les valeurs tolérées par la norme du Codex Alimentarius (0,01 ppm). Les laits de Kpinnou et d'Adjohoun avaient respectivement des taux d'aflatoxine M1 de 0,3 $\pm 0,000$ ppb et 0,24 $\pm 0,010 \mathrm{ppb}$ et n'étaient pas non plus conformes à la norme du Codex Alimentarius 20 (0,05 ppb). Bien que riche en nutriments, le lait de vache analysé présenterait des risques de santé aux consommateurs en raison des contaminants chimiques.

Mots clés : Race bovine, lait, sécurité sanitaire, normes, contaminants chimiques

\section{Introduction}

Dans le cadre de son plan stratégique de relance de son économie, le Bénin à l'instar de nombreux pays de la sous-région accorde un intérêt particulier au secteur de l'élevage et à la production du lait et de ses sousproduits. Selon certains travaux dont ceux de Bawath et Amoussou (1998) et Kora (2005), le nombre de vaches traites chaque année représente environ $15 \%$ de l'effectif du cheptel bovin dans la sous-région ouest-africaine et en particulier au Bénin. Sur cette base, la production laitière du Bénin est passée de 83.000 tonnes en 2003 (MAEP, 2006) à 100000 tonnes environ en 2012 (MAEP, 2013), mais reste encore inférieure aux prévisions de 178582 tonnes faites par Abiola (2007) dans le programme de développement de productions animales en 2007. En effet, la consommation du lait connaît de nos jours un essor considérable, y compris dans les régions côtières caractérisées par un élevage bovin faiblement développé (Dossou et al., 
2006).Le lait contribue à plus de 50\% aux revenus annuels des ménages peulh (Ogodja et al., 1991) et reste un produit très périssable. Ceci constitue l'un des problèmes qui freinent la promotion de la filière lait et produits laitiers au Bénin (Kees 1996). En plus du faible niveau de production nationale du lait, la qualité de cette denrée reste tout aussi préoccupante. Par ailleurs, il faut noter que plusieurs travaux dont celui de Kora (2005) et celui de Sacramento (2008) ont déjà porté sur l’évaluation de la qualité microbiologique et physicochimique du lait de différentes races de vaches. Mais ces derniers n’ont pas porté sur la recherche de germes de zoonoses du genre Brucella et Mycobacterium. Ces germes, pourtant invasifs et toxinogènes, provoquent souvent des lésions au niveau du tractus digestif, mais aussi au niveau d'autres tissus (Cuq, 2007) dans l'organisme humain. Selon Seegers et al., (1997) et Bradley (2002), le risque zoonotique lié à la consommation du lait contaminé par certains germes dangereux est important. Par ailleurs, des études récentes effectuées au Bénin ont révélé que les staphylocoques sont les causes principales des mammites subcliniques dans les élevages bovins laitiers semi intensifs (Kadja, 2010). Ces différents travaux montrent clairement que plusieurs pathologies constituent des contraintes au développement de l'élevage en général et de la production laitière en particulier. Cependant, la recherche des contaminants chimiques du type de métaux lourds (dont le plomb) ou de résidus de pesticides et d'aflatoxine M1 a été épargnée par ces différents travaux récents alors que, le lait concerné ici, consommé frais ou transformé, provient souvent d'un système d'élevage agropastoral extensif, où les vaches laitières se nourrissent aussi bien de fourrage que de résidus agricoles susceptibles d'être contaminés par l'aflatoxine M1. Le bassin laitier béninois, constitué essentiellement des départements du nord (Borgou, Alibori, Atacora et Donga) et du centre (Zou et Collines) coïncide également avec le bassin cotonnier. La culture du coton est associée à une forte utilisation d'intrants polluants et contaminants, notamment les engrais $(\mathrm{N}, \mathrm{P}$, $\mathrm{K}$ ), les pesticides et les herbicides. De même, l'élevage péri-urbain, de nos jours en pleine expansion dans certaines de nos villes, est pratiqué dans des zones de forte pollution atmosphérique par les gaz à effet de serre, de pollution des eaux et des sols par décharges de déchets solides et liquides de toute catégorie. Dans un rapport technique de l'OMS et de la FAO publié en 1972, les plomb-tétra-alcoyles qu'on ajoute à l'essence constituent une source de contamination méritant une attention particulière (FAO/OMS, 1972). Ces différents faits relevés ci-dessus montrent que la consommation du lait pourrait constituer un risque pour la santé du consommateur. C'est dans cette optique que la présente étude s'est fixée pour objectif principal l'analyse de la qualité du lait cru produit par différentes races bovines dans le sud et le centre du Bénin. Pour atteindre cet objectif principal, elle se 
propose d'identifier les différentes races bovines produisant du lait dans le sud et le centre du Bénin, (les races Girolando, Borgou, Borgou-gir et Lagunaire), de faire l'étude comparée de la qualité microbiologique et physicochimique du lait cru produit par ces mêmes races bovines dans cette même zone d'étude, et enfin de comparer le niveau de contamination en plomb, en résidus de pesticides, et en aflatoxine M1 dudit lait.

\section{Matériel et méthodes}

\section{Cadre d'étude}

L’étude a été réalisée dans les localités indiquées sur la figure 1.

La commune d’Adjohoun, située dans le département de l’Ouémé, abrite les vaches de race lagunaire reconnues pour leur faible productivité de lait. Elles produisent en moyenne 0,5 litre de lait par jour et par vache (Dossou et al.,2006). De plus, la proximité de cette commune du fleuve Ouémé (susceptible de drainer les résidus d'engrais chimiques et de pesticides) fait d'elle un point important pour la prise d'échantillons. Les fermes d'élevage de Kpinnou, de Bétécoucou et d’Okpara respectivement dans les communes de Lokossa, de Dassa-Zoumé et de Parakou sont des fermes d'État qui expérimentent l'élevage des principales races bovines au Bénin. La ferme d’élevage de Kpinnou est retenue pour sa situation géographique au Sud du Bénin et aussi pour la présence sur cette ferme des vaches de races importées (Gir et Girolando) très productrices de lait dont la race Girolando d’origine brésilienne est la race d’intérêt pour la présente étude. Elle produit en moyenne 9 litresde lait par jour et par vache dont une grande partie est transformée en fromage (Kora, 2005). La ferme d’élevage de Bétécoucou, située dans la commune de Dassa-Zoumé au centre du Bénin, dispose de troupeaux de vaches de race Borgou qui est une race locale. Cette race de vache produit environ 1 à 2 litres de lait par jour par vache en saison pluvieuse (MAEP/FAO, 2010). De plus, Dassa-Zoumé est un point de forte production de fromage peulh. La matière première servant à cette production de fromage, provient partiellement de la ferme d'élevage de Bétécoucou. Enfin, en ce qui concerne la ferme d'élevage d'Okpara, située dans la commune de Parakou, la présence d'une diversité de races bovines hybrides, à savoir : Borgou-Girolando, Borgou-Hostein, Borgou-Nellore et Borgou-Gir a déterminé le choix de cette ferme. De toutes ces races citées, les hybrides Borgou-Giront été retenues pour l'étude à cause de leur forte production de lait. En effet la vache Borgou-Gir produit environ 10 litres de lait par jour et par vache (MAEP/FAO, 2010). L’approche méthodologique utilisée dans le présent travail comporte l'enquête d'évaluation des différentes mesures d'hygiène prises lors de la traite et de la collecte du lait, les différentes analyses microbiologique, physico-chimique et la recherche de contaminants dans les laits des 
différentes races ciblées. L’enquête s’est adressée aux principaux acteurs de la chaîne de production-vente du lait. Il s’agit des vétérinaires chargés des soins sanitaires des troupeaux, des bouviers chargés de la traite du lait et de conduite de troupeaux au pâturage et des éleveurs propriétaires de troupeaux. L’enquête a permis de connaître les caractéristiques des différentes races bovines identifiées, et les différentes rations alimentaires auxquelles les vaches sont soumises.

\section{Matière première}

La matière première utilisée dans le présent travail est constituée de lait de vache. Il a été prélevé respectivement chez les vaches de race Girolando à la ferme d'élevage de Kpinnou, Borgou à la ferme d'élevage de Bétécoucou, Borgou-Gir à la ferme d’élevage d’Okpara et Lagunaire à Adjohoun.

\section{Matériel de prélèvement du lait}

Le matériel de prélèvement du lait utilisé pour la présente étude est constitué de bouteilles de 1 litre stérilisées, à fermeture hermétique, pour contenir le lait; de gants stériles pour limiter la contamination lors des prises d’échantillons; de papiers en aluminium, pour protéger le couvercle et le col des bouteilles ; de ruban adhésif pour maintenir le papier en aluminium ; de glacières munies de glaçons pour contenir et conserver les échantillons au cours du transport ; l'eau de javel a été utilisée pour nettoyer les mamelles des vaches et enfin l'alcool à $90^{\circ}$ pour nettoyer le matériel non stérile avant et après usage. Un flacon de $5 \mathrm{~L}$ préalablement stérilisé a été utilisé pour le prélèvement du lait.

\section{Identification des troupeaux et collecte des échantillons de lait}

Les troupeaux choisis remplissaient des critères de sélection définis comme suit: ils devraient appartenir à un système d’élevage sédentaire ou semi-transhumant; avoir un effectif d'au moins 50 têtes; avoir un effectif où la race recherchée pour l’étude est représentée; enfin avoir au moins 10 vaches allaitantes. Les vaches retenues pour la traite ne présentaient aucun signe de maladies, étaient déclarées bien portantes par l'agent vétérinaires de la localité et la direction des différentes fermes et dont le lait était classé apte à la consommation humaine. Toutes les vaches allaitantes desdits troupeaux ont été numérotées et 10 d’entre elles ont été tirées au sort et retenues pour la traite. Toutes ces 10 vaches étaient traites à raison de 0,5 litre de lait environ par vache pour obtenir un mélange de 5 litres de lait par race bovine et par localité. La traite a été effectuée en respectant les mesures d'hygiène suivantes: la vache était sortie du troupeau et placée à un endroit assaini, éloigné des fourrages ou autres aliments farineux susceptibles de produire de 
la poussière et de contaminer le lait; les mains et les gants du trayeur étaient rincées avec de l'eau javellisée, avant chaque séance de traite ; le trayon était nettoyé, avant le prélèvement, avec de l'eau javellisée titrée à $0,5 \%$ de chlore actif pour éliminer les fragments de déchets et les salissures adhérant à la peau de la vache ; l'extrémité du trayon est ensuite désinfectée à l'aide de compresses stériles imbibées d'alcool à $70 \%$; après avoir jeté les premiers jets, le lait est recueilli dans un flacon de 5 litres préalablement stérilisé et maintenu incliné de façon à éviter la pénétration des poussières et des poils; le flacon est immédiatement rebouché hermétiquement après que les jets de lait nécessaires soient tirés; les flacons de lait sont identifiés, numérotés puis rassemblés par lots sur lesquels sont mentionnés tous les renseignements nécessaires (nom et prénom du propriétaire, adresse, localité, l’heure de traite, la race bovine, etc.). Après la traite, l'échantillon de 5 litres de lait mélangé et homogénéisé est réparti dans quatre bouteilles stériles de 1 litre, qui sont conservées sous glace dans quatre glacières remplies de glaces et acheminées vers les laboratoires pour les analyses microbiologiques, physico-chimiques et la recherche des contaminants chimiques. Arrivés au laboratoire, les échantillons sont conservés dans un réfrigérateur à une température de $4^{\circ} \mathrm{C}$ pendant 1 à $2 \mathrm{~h}$ avant le démarrage des analyses.

\section{Analyses microbiologiques du lait}

Les analyses microbiologiques effectuées sur les laits ont porté sur la recherche de bacilles de Koch (Mycobacteriumtuberculosis et Mycobacteriumbovis), de Brucella et de mammites. Ainsi, la recherche des germes du genre Mycobacterium a été effectuée par coloration de ZIEHL (Cuq, 2007). Par ailleurs, la recherche de Brucella dans les laits a été effectuée par sérologie (Cuq, 2007). Quant à la recherche d'une contamination du lait par la mammite, une étude cytologique a été effectuée selon la méthode décrite par Cuq (2007).

\section{Analyses physico-chimiques du lait}

Les analyses physico-chimiques du lait ont porté sur le $\mathrm{pH}$, l'acidité titrable, la densité, les teneurs en minéraux $\left(\mathrm{Mg}^{2+}, \mathrm{Ca}^{2+}, \mathrm{K}^{+}, \mathrm{Fe}^{2+}, \mathrm{Zn}^{2+}\right)$, en lactose, en matière sèche, en protéine brute et en matière grasse. Ainsi, le taux de matière sèche a été déterminé par la méthode AOAC (1984). La teneur en protéines brutes a été déterminée par la méthode de Kjeldahl. Le $\mathrm{pH}$ a été mesuré à l'aide d'un $\mathrm{pH}$-mètre numérique de laboratoire de marque Lab 860, préalablement étalonné avec des solutions tampons de $\mathrm{pH} 7$ et 4 à la température de $28^{\circ} \mathrm{C}$. Cette mesure de $\mathrm{pH}$ a été faite sur $25 \mathrm{~mL}$ de l'échantillon. L'acidité titrable a été déterminée par la méthode OIV-MAAS313-01. La densité a été déterminée à l'aide d'un thermo-lactodensimètre. La valeur lue est ramenée à $20^{\circ} \mathrm{C}$ par la formule suivante : densité corrigée $=$ 
densité lue $+0,2$. La teneur en matière grasse a été dosée par la méthode de référence GERBER.Le taux de lactose est déterminé par la méthode de BERTRAND. Les minéraux $\left(\mathrm{Mg}^{2+}, \mathrm{Ca}^{2+}, \mathrm{K}^{+}, \mathrm{Fe}^{2+}, \mathrm{Zn}^{2+}\right)$ ont été dosés par spectrométrie d’absorption atomique. Toutes les analyses ont été répétées trois fois et la moyenne des valeurs obtenues a été considérée.

\section{Analyse des contaminants du lait Recherche des métaux lourds}

L’identification et le dosage des métaux lourds ont été effectués par Spectrométrie Atomique d'Absorption après incinération sèche, selon la méthode NMKL 139 (1991), AOAC 999.11.

\section{Recherche des résidus de pesticides}

L'identification et le dosage des résidus de pesticides ont été effectués par la méthode MET-LFSAL-404. Cette méthode est basée sur le principe selon lequel, l'échantillon est homogénéisé. Ensuite un aliquote est mis au contact d'acétonitrile et une quantité connue de standard interne est ajoutée. L'extraction se fait par sonication et agitation. La purification de l'extrait se fait en trois étapes : centrifugation, congélation du surnageant (élimination des graisses) et passage sur cartouches C18 et d'Al-N. Une phase de concentration par évaporation « sous-vide » termine la préparation de l’échantillon. La solution est injectée sur un système chromatographique gazeux avec détection par spectrométrie de masse. Les pics sont construits sur un des fragments caractéristiques de chaque molécule. La détection des molécules se fait par comparaison des surfaces des pics obtenus avec des matrices vierges dopées à $10 \mu \mathrm{g} / \mathrm{kg}$ et préparées de la même façon que les échantillons inconnus. Il s'agit donc d'une méthode de screening avec calibration interne à un seul niveau de calibration.

\section{Recherche de l'aflatoxine M1}

La recherche d'aflatoxine M1 dans les échantillons de laits par HPLC a été effectuée par la méthode MET-LFSAL-023 par dérivation des aflatoxines M1. Ceci est nécessaire afin d'augmenter leur fluorescence naturelle sous lumière UV, et de pouvoir accroitre la sensibilité du dosage. Le principe de cette méthode est basé sur l'extraction, la purification, le dosage et l'utilisation des colonnes d'immuno-affinité.

\section{Analyses statistiques des données}

L’analyse des données est faite avec le logiciel MINITAB 14. Les méthodes d'analyses utilisées sont: l'analyse de variance (ANOVA) et le test de comparaison de Fisher. Le niveau de significativité a été fixé à 5\%. 


\section{Résultats}

\section{Alimentation et soins des animaux}

Dans les trois fermes d'élevage, deux aliments constituent la ration des animaux: il s'agit du fourrage et des compléments alimentaires. En ce qui concerne le fourrage, les animaux sont conduits deux fois dans la journée au pâturage, de 6 à 10 heures les matins et de 14 à 18 heures les soirs. Ils se nourrissent généralement des espèces telles que Panicum maximum, Brachiaria ruziziensis, Pennisetum purpuriueum et autres. Quant aux compléments alimentaires, ils sont composés de son de riz, de céréales, de tourteaux de coton et de palmiste, d'acides aminés, decalcaire, de phosphate bicalcique et de sel (chlorure de sodium). Par opposition à ce soin alimentaire donné aux animaux de ces différentes fermes, les vaches traites dans la commune d'Adjohoun sont livrées à elles-mêmes et se nourrissent de ce qui s'offre à elles dans le périmètre d'à peine 2 à $3 \mathrm{~m}$ de rayon qui leur est défini par leurs cordes toujours attachées au cou.

Dans les trois fermes enquêtées, les mesures d'hygiènes ont été évaluées sous trois angles différents à savoir, l'hygiène des locaux, l'hygiène corporelle et l'hygiène de traite. Pour l'hygiène des locaux, les agents d'entretien des parcs ramassent quotidiennement les bouses de vache; l'étable est nettoyée régulièrement avec de l'eau simple sans être désinfectée avec du crésyl. Ensuite pour l'hygiène corporelle, le troupeau bénéficie d'un bain constitué de l'eau et du Nokalt (produit vétérinaire) pour lutter contre les tiques et autres insectes. Enfin, les mains des trayeurs, les seaux et bonbonnes de collecte sont soigneusement nettoyés avant la traite du lait. Par ailleurs, la traite n'étant pas habituellement pratiquée par les éleveurs de vaches lagunaires d'Adjohoun, aucune règle d'hygiène n'est accordée aux animaux. De plus l'intervention du vétérinaire est sollicitée par les éleveurs seulement en cas de force majeure.

\section{Qualité microbiologique des laits}

Tous les échantillons de lait de vache de race Girolando, Borgou, Borgou-Gir et Lagunaire sont exempts d'affections dues au Brucella, au Mycobacterium et à la mammite.

\section{Caractéristiques physico-chimiques du lait}

Le tableau 1 présente les caractéristiques physico-chimiques du lait des vaches de race Girolando, Borgou, Borgou-Gir et Lagunaire dont les échantillons sont pris respectivement sur les fermes deKpinnou, Bétécoucou, Okpara et dans la commune d'Adjohoun. Ces résultats montrent que le $\mathrm{pH}$ de ces différents laits varie entre 6,5 $\pm 0,000$ et 6,8 $\pm 0,057$. Les $\mathrm{pH}$ des laits de vache de race Borgou et Lagunaire sont identiques d'une part et d'autre part, ceux des laits de vache de race Girolando et Borgou-Gir le sont aussi. 
Quant à l'acidité titrable, une différence significative $(\mathrm{p}<0,05)$ a été observée entre tous les laits des différentes races de vaches. La teneur en matière sèche des laits de vache des races étudiées varie entre 12,89\% et 13,2 \%. Cette teneur est identique pour les laits de vache des races Girolando et Lagunaire tandis que les teneurs en matières sèches des laits de vache des races Borgou et Borgou-Gir sont différentes. En ce qui concerne la température de congélation, une différence significative $(\mathrm{p}<0,05)$ entre les laits des vaches a été constatée. La densité à $20^{\circ} \mathrm{C}$ de ces différents laits varie entre 1,028 $\pm 0,002 \mathrm{~g} / \mathrm{cm}^{3}$ et $1,032 \pm 0,002 \mathrm{~g} / \mathrm{cm}^{3}$. Le tableau 2 présente les éléments nutritifs du lait des vaches des races étudiées des quatre localités. Les analyses statistiques ont montré qu'il n’y a aucune différence significative $(p=0,673)$ entre les teneurs en matière grasse des laits des différentes races bovines étudiées. Mais pour ce qui est de la teneur en lactose, elle a varié entre 4,48 $\pm 0,011$ et 4,67 $\pm 0,010 \mathrm{~g} / 100 \mathrm{~g}$ de MS. Les laits des vaches des races Borgou et Lagunaire présentent les mêmes teneurs en lactose alors que celles des laits des races Girolando et Borgou-Gir sont différentes. En ce qui concerne la teneur en protéine et en minéraux (Fer, Zinc, Magnésium, Potassium, Calcium), une différence significative est observée entre les laits des différentes races bovines. Globalement, les teneurs en protéines et minéraux les plus élevées ont été observées au niveau du lait de la race Borgou-Gir alors que les faibles teneurs en ces différents éléments ont été respectivement observées au niveau des laits des vaches des races Lagunaire et Girolando. Le tableau 3 présente les résultats statistiques obtenus après la recherche de contaminants dans les laits. Les analyses ont révélé que tous les laits de vache des différentes races étudiées contiennent des résidus de plomb dans des proportions variant de 0,0204 $\pm 0,000$ à 0,0490 $\pm 0,001 \mathrm{ppm}$. Ces teneurs dépassent la valeur maximale prévue par la norme Codex Standard 193-1995 qui est de 0,02 ppm. Les laits des vaches de races Girolando et Lagunaire n’ont contenu aucune trace de résidus de pesticides. Par contre, les laits des vaches de races Borgou et Borgou-Gir ont été contaminés par les résidus de pesticides dans des proportions dépassant la norme de 0,01 ppm admise par le Codex Standard 193-1995. Les teneurs en aflatoxine M1 variant entre $0,24 \pm 0,010$ et $0,3 \pm 0,000$ ppb ont été observées respectivement pour les laits des vaches de race Lagunaire et Girolando. Ces teneurs observées dépassent de la limite maximale de 0,05 ppb de lait prévue par la norme Codex Standard 193-1995.

\section{Discussion}

Interprétations des caractéristiques physicochimiques et nutritionnelles des laits

L’évaluation de la qualité physicochimique et nutritionnelle montre que les laits des différentes races présentent des caractéristiques 
satisfaisantes en ce qui concerne le pH, l’acidité titrable, la densité, la température de congélation et les teneurs en matière sèche, en matière grasse, en magnésium, en potassium, en zinc et en lactose. En effet, le $\mathrm{pH}$ des différents laits étudiés varie entre 6,5 \pm 0,000 et 6,8 \pm 0,057 et est conforme aux valeurs obtenues par Kora (2005) et Caset (2007). Pour Caset, le pH du lait varie entre 6,5 et 6,7, ne change théoriquement pas et constitue un indice de son état de fraîcheur. Les valeurs du $\mathrm{pH}$ obtenues dans le cadre de cette étude pourraient être dues à la conservation de l'état de fraicheur des laits au cours de leur transport. Car selon Caset (2007), lors d'un manque de fraîcheur, les bactéries lactiques transforment le lactose en acide lactique qui diminue le $\mathrm{pH}$ du lait par augmentation de la concentration d'ions hydronium $\mathrm{H}_{3} \mathrm{O}^{+}$. Selon Wattiaux (1997), la densité du lait varie de 1,023 à 1,040 et évolue dans le même sens que la matière sèche tel que le présentent les valeurs obtenues pour cette étude. Les différents laits ont une température de congélation comprise entre $-0,550^{\circ} \mathrm{C}$ et $-0,520^{\circ} \mathrm{C}$. Ce qui montre que les échantillons de laits utilisés sont issus de la traite directe des vaches et n’ont subi aucune adjonction d'eau. Pour Luquet (1985) et Wattiaux (1997), la température de congélation varie entre $-0,51$ et $-0,55^{\circ} \mathrm{C}$, selon les conditions zootechniques. Si elle tend vers $0^{\circ} \mathrm{C}$, cela peut permettre de détecter une adjonction d'eau ou mouillage, qui constitue une fraude (Luquet, 1985; Wattiaux, 1997). Il ressort des résultats de cette étude que les teneurs en fer et en calcium de tous les laits étudiés sont très élevées comparativement aux valeurs obtenues par Caset (2007). Quant à la teneur en protéine, elle est aussi très élevée pour tous les laits comparativement aux valeurs obtenues par Kora (2005). Ces différents laits étudiés sont alors très riches en protéine, en fer et en calcium. Ils présentent des teneurs similaires à celles de Caset (2007) en ce qui concerne les teneurs en matière sèche, en matière grasse, en lactose, en magnésium, en potassium et en zinc. Par ailleurs, le lait obtenu des vaches de race Borgou-Gir est le plus nutritif de par sa forte teneur en lactose, en protéine, en magnésium, en calcium, et en zinc. Il sera un bon aliment pour la petite enfance, l'enfance et l'adolescence. Il constituera également une matière première idéale pour la transformation en produits laitiers dont le fromage traditionnel peulh « wagashi ».

\section{Les contaminants des laits}

Tous les laits contiennent des résidus de plomb dont les teneurs dépassent les prévisions de la norme du Codex Standard 193-1995. Selon Oskarsson et al. (1998) et Swarup et al. (1997), les principales sources de contamination du lait par le plomb sont d'origine industrielle, notamment la manipulation de peinture, d'essence ou de batteries. De plus, dans leur rapport technique publié en 1972, la FAO et l'OMS montrent que la présence possible de résidus de plomb dans le lait provient de trois sources de 
contamination possibles : l'air, l'alimentation et l'eau (FAO/OMS, 1972). Ainsi, cette pollution des laits par le plomb pourrait être due à la proximité des fermes des grandes agglomérations. Ce qui entrainerait une pollution de l'eau d'abreuvage et des pâturages par les gaz d'échappement. En effet, les plomb-tétra-alcoyles qu'on ajoute à l'essence constituent une source de contamination en ce métal lourd (FAO/OMS, 1972). Ces produits augmentent non seulement la quantité de plomb dans l'air inhalé par les vaches allaitantes, mais aussi l'apport par ingestion, dans la mesure où les retombées des gaz d'échappement se déposant sur les récoltes et dans l'eau d'abreuvage des vaches sont importantes et varient selon le degré d'urbanisation, la situation topographique et les conditions atmosphériques (FAO/OMS, 1972). La consommation de ces laits contaminés constitue un danger pour le consommateur car selon Vidal (2008), l'accumulation des résidus de plomb dans l'organisme par le biais de la consommation des laits pourrait provoquer plusieurs maladies parmi lesquelles on peut citer l'autisme et les maladies neurodégénératives, mais aussi des troubles mineurs comme les troubles du comportement chez l'adulte et l'enfant, les troubles du sommeil, les troubles digestifs, les maux de tête, les dépressions légères, les problèmes de peau, etc. (Vidal, 2008). De plus, une plombémie élevée peut être à l'origine de plusieurs maux tels que: le syndrome abdominal, l’encéphalopathie, les neuropathies périphériques, les atteintes rénales, l'hypertension artérielle et l'hyper-uricémie. Le plomb peut également être à l'origine des avortements spontanés chez des femmes qui y sont exposées pendant leur grossesse (Bismuth et al., 2002). Contrairement aux laits des vaches de races Borgou et Borgou-Gir, les laits de vache des races Girolando et Lagunaire contiennent de l'aflatoxine M1 dont les teneurs sont supérieures à la limite maximale de résidus d'aflatoxine M1 dans le lait, exigée par la norme Codex Standard 193-1995. La présence d'aflatoxine M1 dans les laits de ces races de vache peut être due aux conditions de stockage des produits constituant le régime alimentaire des vaches de ces races. En effet, selon Cuq (2007), l'aflatoxine peut être produite dans des graines (riz, soja, coton, arachide, blé, etc.) et dans les produits dont la teneur en eau est supérieure à $15-17 \%$. De plus, les vaches de race Girolando étant des races d'origine étrangère, elles peuvent être plus sensibles aux attaques microbiennes à cause du changement de leur milieu de vie naturel. Pour ce qui est de la race Lagunaire, les vaches de cette race sont élevées en divagation, donc livrées à elles-mêmes et se nourrissent de résidus de récolte dans les champs de maïs, de soja et d'arachide. Ce régime alimentaire des vaches de cette race pourrait être la cause de la contamination de leur lait. Ces laits contaminés par l'aflatoxine M1 peuvent être une source d’intoxication sérieuse pour les consommateurs car selon Moreau (1976), Gallotti et Fremy (2006), Rouillé et Thual (2011), les maladies dues aux 
aflatoxines atteignent principalement les enfants de 3 à 8 ans et une intoxication aigüe se manifeste par une hépatite aigüe, le kwashiorkor ou le syndrome de Reye. D’après ces mêmes auteurs, une exposition chronique à l'aflatoxine serait liée à l'apparition du cancer primitif du foie et est considéré comme cancérigène possible pour l’homme. Cependant, de tous les laits, seuls ceux des vaches de race Borgou et Borgou-Gir contiennent des résidus de pesticides (Chlorpyriphos-éthyl et Cyperméthrine). La teneur en

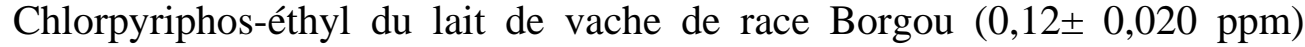
dépasse la limite maximale de résidus de ce principe actif dans le lait qui est de 0,01 ppm. Par contre le lait de vache de race Borgou-Gir contient 0,03 \pm 0,020 ppm de Cyperméthrine, une teneur inférieure à la limitemaximale de résidus de Cyperméthrine dans le lait (0,05 ppm). Selon Adib et Bertrand (2009) et Rychen et al. (2005), les résidus de pesticides pénètrent dans l'organisme des vaches par voie cutanée, par inhalation et surtout par voie digestive, cette dernière intervenant de façon prépondérante. Les vaches sont exposées à trois sources de contamination majeures responsables de la présence possible de résidus de produits phytosanitaires dans le lait: il s’agit de l'alimentation, des bâtiments d'élevage et de l'usage de produits antiparasitaires externes (Adib et Bertrand, 2009). Ainsi, le traitement des semences ou des parties aériennes des plantes cultivées, destinées à l'alimentation des vaches laitières et l'assainissement des locaux d'élevage et le traitement antiparasitaires des vaches laitières dans les Fermes d'Élevage de Bétécoucou et de l'Okpara pourraient être à l'origine de la contamination de ces laits par le chlorpyriphos-éthyl et le cyperméthrine. De plus, l'ingestion de terre et d'eau contaminées par des résidus de pesticides (l'eau du fleuve Okpara à proximité de la ferme d'élevage de l'Okpara et l'eau du fleuve Ouémé à proximité de la ferme de Bétécoucou) pourrait être à l'origine de cette contamination des laits de races Borgou et Borgou-Gir. Rychen et al. (2005) estiment que c'est par l'intermédiaire de la consommation de végétaux dont les racines puisent dans le sol, par ingestion de terre quand les vaches broutent et par la contamination des nappes phréatiques dont l'eau peut ensuite être ingérée par les ruminants que la contamination peut se produire. Chez les personnes très exposées comme les agriculteurs et les consommateurs, lorsque la limite maximale de résidus (LMR) est atteinte, les pesticides multiplient les risques de maladie de Parkinson, de cancer de la prostate et de certains cancers hématopoïétiques (lymphome non Hodgkinien, myélomes multiples). Par ailleurs, les expositions aux pesticides intervenant au cours de la période prénatale et périnatale ainsi que de la petite enfance semblent être particulièrement à risque pour le développement de l'enfant (INSERM, 2013).

\section{Interprétations des résultats issus des analyses microbiologiques}


Cette étude, portée sur la recherche des principales maladies liées à la production laitière, a visé trois pathologies, qui, selon Traoré et al. (2004) ont une grande importance sur le plan économique et sanitaire. Il s’agit de la brucellose, la tuberculose et les mammites. Ces trois pathologies sont absentes dans tous les laits de vache des différentes races étudiées. Cette absence de contamination des laits par Brucella reflète la situation au niveau national en ce qui concerne cette affection. En effet, d'après les rapports annuels de l'Office International des Epizooties (OIE), de l'Organisation des Nations Unies pour l'Alimentation et l'Agriculture (FAO) et de l'Organisation Mondiale de la Santé (OMS) de 1974, la présence de la brucellose bovine au Bénin est soupçonnée, mais non confirmée jusqu'en 1974 (Chantal et Ferney, 1976). Des études menées plus tard, ont montré qu'au Bénin,le taux moyen d'infection brucellique des bovins est de 10,4 \% (Acakpo et Bonarel, 1987). Mais les études réalisées par le Laboratoire de diagnostic vétérinaire de Bohicon (2000) dans les quatre fermes d'Etat du Bénin ont donné des taux d'infection brucelliques de $0 \%$ pour les fermes de Bétécoucou et de Samiondji, 2,5\% à Kpinnou et 2,15\% à la ferme de l'Okpara. Pour Koutinhouin et al. (2003), un test sérologique de dépistage de la brucellose dans une population conduit forcement à des résultats positifs, mais rien ne permet de dire qu'il s’agit effectivement de brucellose ; et par ricochet de confirmer une contamination du lait par la mammite. Car, d'après Roguinsky et al., (1972), la présence de Brucella provoque une mammite brucellique subclinique. Toutefois, la relation entre l'infection de la carcasse et la présence de Brucella dans le lait n'est pas absolue (Philippon, et al., 1971). Par ailleurs, Fadrig (1988); Millet (1988) et Spencer (1992) ont montré que la présence de ces infections mammaires pourrait être attribuée aux mauvaises conditions d'hygiène de la traite qui favorisent la transmission de l'infection d'un quartier à un autre ou d'une vache à une autre. De plus, bien plus tard, Boussini et al. (2012) ont confirmé à leur tour que le système d'élevage, l'âge et le sexe de la vache ont un effet statistiquement significatif sur la prévalence de la tuberculose, contrairement à la brucellose pour laquelle seul le système d'élevage aurait une influence significative. Quant à AFSCA (2013), les bovins sont généralement contaminés par l'ingestion d'aliments contaminés ou en buvant de l'eau contaminée. Ceci pourrait justifier les résultats de cette étude qui s’est effectuée sur des fermes d'élevage où la traite se fait dans le respect des conditions d'hygiène.

\section{Conclusion}

La présente étude, menée dans quelques élevages de la commune d’Adjohounet sur les fermes d’élevages de Kpinnou, Bétécoucou et Okpara, a pu évaluer quelques caractéristiques microbiologiques, physico-chimiques 
et quelques contaminants du lait de quatre races de vaches Girolando, Borgou-Gir et enfin les races Borgou et Lagunaire. Des analyses effectuées sur les échantillons de lait, il ressort que le lait de vache de race Borgou-Gir dont les échantillons sont prélevés sur la ferme d’élevage d’Okpara est la plus riche en eau, lactose, protéine, zinc, magnésium et calcium. Ce lait ne présente aucune contamination par les résidus de pesticides, d'aflatoxine M1, ni aucune affection à la brucellose, à la tuberculose et à la mammite. Par contre, on y note la présence de plomb dont la teneur dépasse la limite maximale autorisée. La consommation de ce lait ou de ses produits dérivés représente un risque pour la santé des consommateurs. Par ailleurs, le lait de vache de race Lagunaire dont les échantillons sont prélevés dans la commune d'Adjohoun est la plus riche en matière grasse et en potassium. Pour cette race, la densité et la température de congélation sont également plus élevées. Afin de mieux connaître la qualité sanitaire du lait produit au Bénin, il est nécessaire d'étendre l'étude sur d'autres races bovines et dans les autres régions du Bénin et de prendre des mesures hardies pour réduire la contamination du lait en aflatoxine, plomb et résidus de pesticides.

\section{References:}

Abiola, F. A. 2007. Programme de développement de l’élevage pour les cinq prochaines années (2007-2012).Rapport de Consultation, 243p.

Adib, A. Bertrand, S. 2009. Rapport du Centre National Interprofessionnel de l'Economie Laitière et de l'Institut de l'Élevage : Analyse des risques de transferts de produits phytosanitaires vers le lait. 13p.

Acakpo, A. J., Bonarel, P. 1987. Epidémiologie des brucelloses animales en Afrique tropicale : enquêtes clinique, sérologique et bactériologique. Revue Sci. Tech. Off. Int. Epiz. 5 : 981-1027. Amoussou, A. R. et Bawath, O. 1998. Etude bilan sur la fromagerie traditionnelle ; Possibilités et limites de la création d'une unité de fabrication artisanale de fromage. Rapport de consultation, MDR, Cotonou.134 p.

AFSCA, 2013. Brucellose, communication de l'Agence Fédérale pour la Sécurité de la Chaîne Alimentaire.

http://www.favv-afsca.fgov.be/dierengezondheid/brucellose/

AOAC 1984. Association of Official Agricultural Chemists. Official Methods of Analysis 10th éd. Washington, D.C., U.S.A

Baldi, I., Brahim, B., Brochard, P., Dartigues, J. F., Salamon, R. 1998. Effets retardés des pesticides sur la santé : état des connaissances épidémiologiques. Rev. Epidémio. Sant. Pub. 46 : 134-142.

Bawath, O. Amoussou, A. R. 1998: Etude-bilan sur la fromagerie traditionnelle. Possibilités et limites de création d'une unité de fabrication artisanale de fromage. Rapport d'enquête PADSA, 134 p. 
Bismuth, C., Baud, F., Conso, F., Dally, S., Fréjaville, J.-P., Garnier, R., Jaeger, A. 2002. Les effets du plomb sur la santé : Toxicologie clinique. Flammarion Médecine-Sciences, 5ème éd. Paris, 1092 p.

Boussini, H., Traoré, A., Tamboura, H. H., Bessin, R., Boly H., Ouédraogo, A. 2012. Prévalence de la tuberculose et de la brucellose dans les élevages bovins laitiers intra-urbains et périurbains de la ville de Ouagadougou au Burkina Faso. Rev. Sci. Tech. Off. Int. Epiz. 31: 943-951.

Bradley, A. J. 2002. Bovine mastitis: an evolving disease. The Vet. J. 164 : 116-128.

Cazet, L. D. M. 2007. Bilan du taux de contamination et étude préparatoire au dosage de résidus de produits phytosanitaires dans le lait de grand mélange bovin. Thèse pour l'obtention du doctorat en médecine vétérinaire ; Université Claude-Bernard - Lyon I.

Chantal, J. et Ferney, J. 1976. La Brucellose bovine en Afrique tropicale : quelques aspects cliniques et épidémiologiques. Rev. Méd. Vét. 127 : 19-42

Cuq, J. L. 2007. Microbiologie alimentaire : Contrôle microbiologique des aliments ; Manuel technique. Université Montpellier 2, 119 pp.

Dossou, J., Adote, S. and Soulé, H. 2006. Fiche technique de production et transformation du lait frais en fromage peulh au Bénin. Guide de Bonnes Pratiques 33 pp.

Fadrig, A. 1988. Contribution à l'étude d'un programme antimammite dans six élevages laitiers de la SODEA., Thèse Doct. Vét., IAV Hassan II, Rabat, Maroc, 137 p.

FAO/OMS 1972. OMS, série de rapports techniques N505 ; Réunions de la FAO sur la nutrition $\mathrm{N}^{\circ} 51$ : Évaluation de certains additifs alimentaires et des contaminants : Mercure, Plomb et Cadmium, 17 p.

FAO/OMS 1995. Norme générale codex pour les contaminants et les toxines présents dans les produits de consommation humaine et animale. Codex Stan 193. 43p.

FAO/OMS-Codex Alimentarius, 2000. Résidus de pesticides dans les produits destinés à l'alimentation Humaine et Animale, 25 p.

Gallotti, S. et Fremy J. M. 2006. Évaluation des risques liés à la présence de mycotoxines dans les chaînes alimentaires humaine et animale ; Rapport final de l’Agence Française de Sécurité Sanitaire des Aliments, 14-17

INSERM 2013. Rapport de l'INSERM, Pesticides : Effets sur la santé, une expertise collective de l'Institut National de la santé et de la Recherche Médical.

Kadja, M. 2010. Étude des mammites subclinques dans les élevages bovins laitiers en Afrique de l’Ouest: cas du Sénégal et du Bénin. Thèse, Université d’Abomey-Calavi, Bénin, 184 p. 
Kees, M. 1996. Le fromage peulh : facile à produire et bien apprécié, une technologie à vulgariser. Rapport de recherche GTZ, Université Eschborn, RFA. pp8-25.

Kodio, A. 2005. Qualité de produits laitiers de production industrielle et artisanale, Thèse de doctorat en pharmacie, Université du Mali, 100 pp.

Kora, S. 2005. Contribution à l'amélioration technologique de fabrication du fromage peuhl : mise au point et test de performance d'un coagulant végétal à base de Calotropisprocera, Thèse d'ingénieur agronome ; Université d'Abomey-Calavi, Bénin, $105 \mathrm{p}$

Koutinhouin, B., Youssao, A. K. I., Houehou, A .E. et Agbadje, P. M. 2003. Prévalence de la brucellose bovine dans les élevages traditionnels encadrés par le Projet pour le Développement de l'Elevage (PDE) au Bénin ; Revue Méd. Vét. 154 :271-276. Laboratoire de diagnostic vétérinaire, Bénin. Rapport annuel d'activité 2000. Ministère du développement rural Cotonou, $61 \mathrm{p}$.

Luquet, F. M. 1985. Laits et produits laitiers : vache, brebis, chèvre. Vol 1. Les laits : de la mamelle à la laiterie.-Paris : Lavoisier 398p.

MAEP 2006. Annuaire statistique du Ministère de l'Agriculture de l'Elevage et de la Pêche ; $31 \mathrm{pp}$.

MAEP/FAO 2010. Rapport, Projet " Appui à l'Amélioration de la Collecte, de la Transformation et de la Commercialisation du Lait, du " wagashi " et autres Produits Laitiers ».

MAEP 2013. Annuaire statistique du Ministère de l'Agriculture de l'Elevage et de la Pêche ; pp.34.

Millet, V. 1988. Mammites : Attention danger! Rev. Fr. Génét. Reprod. 50 : 42-44.

Moreau, C. 1976. Les mycotoxines dans les produits laitiers 298 pp.

Ogodja, J. O., Hounsou-Ve, G., Dehoux, J. P. 1991. Rôle et activité de la femme peulh dans son ménage dans le Sud Borgou au Bénin. Part1.

Oskarsson, A., Hallen, I. P., Sundberg, J., Grawe, K. P. 1998. Risk assessment in relation to neonatal metal exposure. Analyst 123: 19-23

Philippon, A., Renouy, G., Plommet, M. 1971. Brucellose bovine expérimentale V - excrétion de «Brucella abortus » par le colostrum et le lait ; Ann. Rech. Vét. 2 : 9 -67.

Roguinsky, M., Fensterbank, R., Philippon, A. 1972. Influence de l'infection brucellique de la mamelle sur la teneur en cellules du lait ; Ann. Rech. Vét. 3 : 449-457.

Rouillé, B. et Thual, J. 2011. Fiche sanitaire des coproduits : Aflatoxine B1/M1 (AF B1/M1)

Rychen, G., Ducoulombier-Crepineau, C., Grova, N., Jurjanz, S., Feidt, C. 2005. Modalités et risques de transfert des polluants organiques persistants vers le lait, INRA Prod. Anim. 18 : 355-366. 
Sacramento, O. 2008. Contribution à l'amélioration de la conservation du fromage peulh par l'effet combiné du séchage et de l'emballage sous vide. Thèse d'ingénieur agronome; Université d'Abomey-Calavi,Bénin, 74 p.

Seegers, H., Menard, J. L., Fourichon, C. 1997. Mammites en élevage bovin laitier : importance actuelle, épidémiologie et plans de prévention. Rencontres Rech. Ruminants 4 : 233-242.

Spencer, S. B., 1992. Milking machine function and cleaning. In Wilcox, C. J., Van Horn, H. H. (Eds). Large dairy herd management, Gainesville, Florida, 16-19 February Champaign, USA: American Dairy Science Association, 475-486p.

Swarup, D., Dwivedi, S. K., Dey, S. 1997. Lead and cadmium levels in blood and milk of cows from Kanpur city, Indian J. Anim. Sci. 67: 222-223. Traoré, A., Tamboura, H. H., Bayala, B., Rouamba, D. W., Yaméogo, N., Sanou, M. 2004. Prévalence globale des pathologies majeures liées à la production laitière bovine en système d'élevage intra-urbain à Hamdallaye(Ouagadougou) ; Biotechnol. Agron. Soc. Environ. 8 : 3-8.

Vidal V. 2008. Métaux lourds : quelles conséquences sur notre santé?, http://www.valerievidal.fr

Wattiaux, M. A. 1997. Dairy essentials (1st edition): Lactation and milking, The Babcock Publications, University of Wisconsin-Madison, 73-100 pp.

Tableau I : Caractéristiques physico-chimiques des laits des différentes races bovines étudiées

\begin{tabular}{ccccc}
\hline Paramètres recherchés & LVRG & LVRB & LVRBG & LVRL \\
\hline Acidité titrable $\left({ }^{\circ} \mathrm{D}\right)$ & $15,76 \pm 0,020^{\mathrm{a}}$ & $15,82 \pm 0,000^{\mathrm{b}}$ & $16,30 \pm 0,006^{\mathrm{c}}$ & $16,20 \pm 0,000^{\mathrm{d}}$ \\
Densité à $20^{\circ} \mathrm{C}\left(\mathrm{g} / \mathrm{Cm}^{3}\right)$ & $1,032 \pm 0,000^{\mathrm{acd}}$ & $1,030 \pm 0,001^{\mathrm{ac}}$ & $1,028 \pm 0,002^{\mathrm{bce}}$ & $1,032 \pm 0,002^{\mathrm{a}}$ \\
$\mathrm{pH}$ & $6,7 \pm 0,1^{\mathrm{b}}$ & $6,5 \pm 0,057^{\mathrm{a}}$ & $6,8 \pm 0,057^{\mathrm{b}}$ & $6,5 \pm 0,000^{\mathrm{a}}$ \\
Matière sèche $(\% \mathrm{MS})$ & $12,89 \pm 0,010^{\mathrm{a}}$ & $13,2 \pm 0,010^{\mathrm{b}}$ & $13,13 \pm 0,005^{\mathrm{c}}$ & $12,89 \pm 0,000^{\mathrm{a}}$ \\
Température de & $-0,525 \pm 0,001^{\mathrm{a}}$ & $-0,540 \pm 0,000^{\mathrm{b}}$ & $-0,550 \pm 0,004^{\mathrm{c}}$ & $-0,520 \pm 0,000^{\mathrm{d}}$ \\
congélation $\left({ }^{\circ} \mathrm{C}\right)$ & & & &
\end{tabular}

LVRG : Lait de vache de race Girolando ; LVRB : Lait de vache de race Borgou ; LVRBG : Lait de vache de race Borgou-Gir ; LVRL : Lait de vache de race Lagunaire NB : Les valeurs moyennes portant la même lettre sur la même ligne ne sont pas significativement différentes au seuil de $5 \%$.

Tableau II: Les éléments nutritifs du lait

\begin{tabular}{ccccc}
\hline Éléments nutritifs & LVRG & LVRB & LVRBG & LVRL \\
\hline Matière grasse (\%MS) & $3,68 \pm 0,005^{\mathrm{a}}$ & $3,59 \pm 0,100^{\mathrm{a}}$ & $3,60 \pm 0,200^{\mathrm{a}}$ & $3,68 \pm 0,010^{\mathrm{a}}$ \\
Lactose (\% MS) & $4,6 \pm 0,000^{\mathrm{b}}$ & $4,48 \pm 0,011^{\mathrm{a}}$ & $4,67 \pm 0,010^{\mathrm{c}}$ & $4,5 \pm 0,010^{\mathrm{a}}$
\end{tabular}




\begin{tabular}{cccccc}
\multicolumn{2}{c}{ Protéine (\% MS) } & $43,57 \pm 0,000^{\mathrm{a}}$ & $42,70 \pm 0,002^{\mathrm{b}}$ & $63,87 \pm 0,001^{\mathrm{c}}$ & $37,45 \pm 0,010^{\mathrm{d}}$ \\
& Magnésium & 128,053 & $159,047 \pm$ & $197,556 \pm$ & 192,747 \\
& & $\pm 0,002^{\mathrm{a}}$ & $0,003^{\mathrm{b}}$ & $0,001^{\mathrm{c}}$ & $\pm 0,001^{\mathrm{d}}$ \\
Sels & Calcium & 1623,67 & $1561,60 \pm$ & & 1641,29 \\
Minéraux & Fer & $1,732 \pm 0,000^{\mathrm{a}}$ & $0,000^{\mathrm{b}}$ & $2056,32 \pm 0,000^{\mathrm{c}}$ & $\pm 0,000^{\mathrm{a}}$ \\
$($ ppm) & Zinc & $3,715 \pm 0,000^{\mathrm{a}}$ & $2,906 \pm 0,002^{\mathrm{b}}$ & $1,081 \pm 0,001^{\mathrm{c}}$ & $1,728 \pm 0,001^{\mathrm{d}}$ \\
& Potassium & $1169,96 \pm 0,000^{\mathrm{a}}$ & $1293,50 \pm$ & $1290,000^{\mathrm{c}}$ & $3,681 \pm 0,001^{\mathrm{d}}$ \\
& & & $0,000^{\mathrm{b}}$ & $1294,02 \pm 0,000^{\mathrm{c}}$ & $1391,11 \pm 0,000^{\mathrm{d}}$ \\
\hline
\end{tabular}

LVRG : Lait de vache de race Girolando ; LVRB : Lait de vache de race Borgou ;

LVRBG : Lait de vache de race Borgou-Gir ; LVRL : Lait de vache de race Lagunaire

NB : Les valeurs moyennes portant la même lettre sur la même ligne ne sont pas significativement différentes au seuil de 5\%.

Tableau III : Contaminants et toxines du lait

\begin{tabular}{cccccc}
\hline Contaminants & LVRG & LVRB & LVRBG & LVRL & $\begin{array}{c}\text { Norme Codex } \\
\text { Alimentarius }\end{array}$ \\
\hline Plomb (ppm) & $0,0293 \pm 0,000^{\mathrm{a}}$ & $\begin{array}{c}0,0454 \pm \\
0,000^{\mathrm{b}}\end{array}$ & $\begin{array}{c}0,0490 \pm \\
0,001^{\mathrm{c}}\end{array}$ & $\begin{array}{c}0,0204 \pm \\
0,000^{\mathrm{d}}\end{array}$ & $0,02 \mathrm{ppm}$ \\
$\begin{array}{c}\text { Résidus de } \\
\text { pesticide } \\
\text { (ppm) }\end{array}$ & $0 \pm 0,000^{\mathrm{a}}$ & $\begin{array}{c}0,12 \pm 0,020^{\mathrm{b}} \\
\text { (Chlorpyriphos-éthyl) }\end{array}$ & $\begin{array}{c}0,03 \pm \\
0,020^{\mathrm{b}} \\
(\text { Cyperméthrine) }\end{array}$ & $0 \pm 0,000^{\mathrm{a}}$ & $\begin{array}{c}0,01 \mathrm{ppm} \\
\text { (Chlorpyriphos-éthyl) }\end{array}$ \\
$\begin{array}{c}\text { Aflatoxine } \\
\mathrm{M}_{1}(\mathrm{ppb})\end{array}$ & $0,3 \pm 0,000^{\mathrm{a}}$ & $0 \pm 0,000^{\mathrm{b}}$ & $0 \pm 0,000^{\mathrm{b}}$ & $0,24 \pm 0,010^{\mathrm{c}}$ & $0,05 \mathrm{ppp}$ \\
(Cyperméthrine)
\end{tabular}

LVRG : Lait de vache de race Girolando ; LVRB : Lait de vache de race Borgou ; LVRBG : Lait de vache de race Borgou-Gir ; LVRL : Lait de vache de race Lagunaire NB : Les valeurs moyennes portant la même lettre sur la même ligne ne sont pas significativement différentes au seuil de 0,05. 


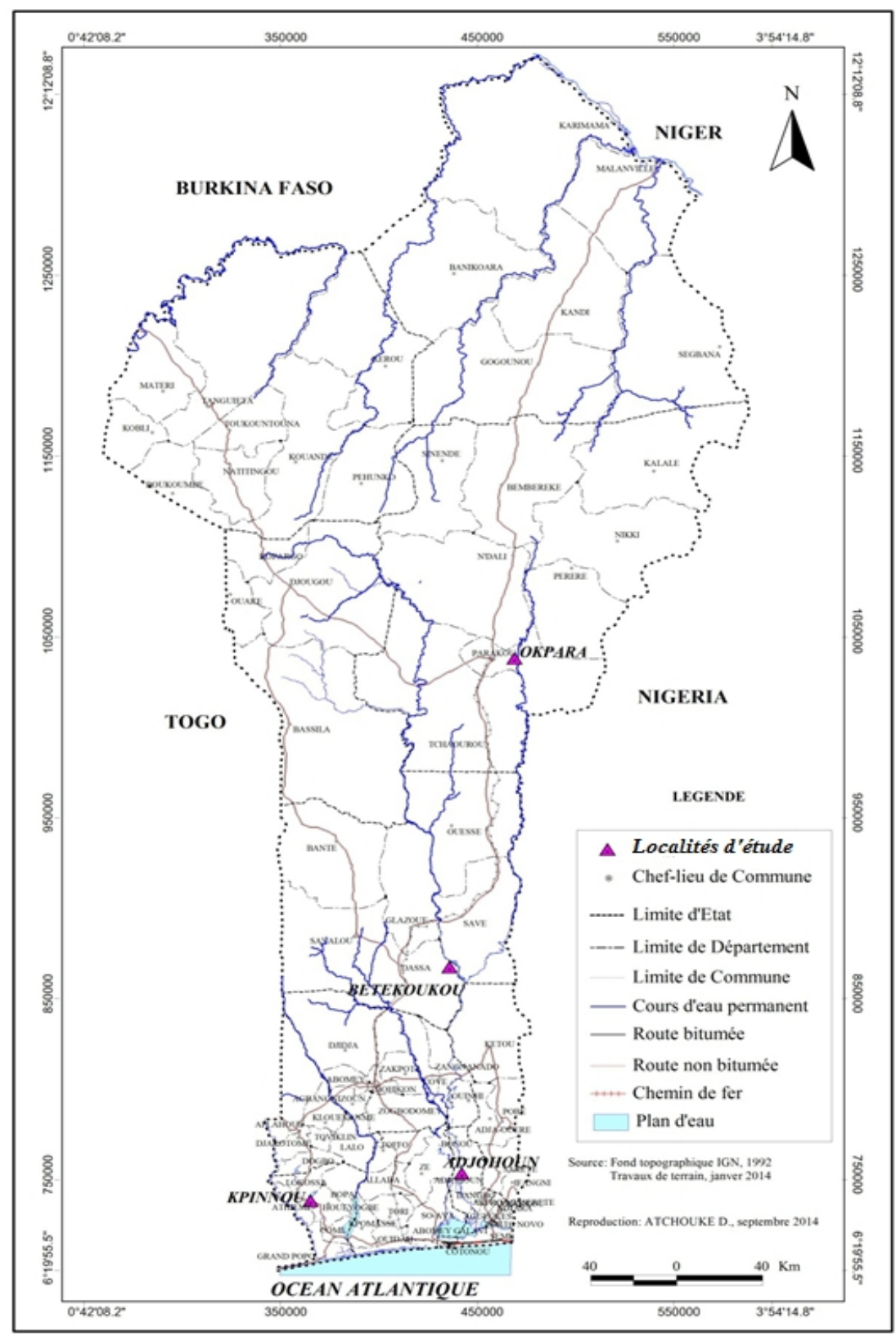

Figure 1 : Situation des zones d'études 